\title{
Mapping of wave packets in direct fragmentation via pump-probe frequency integrated fluorescence spectroscopy
}

\section{Engel, Volker; Henriksen, Niels Engholm}

Published in:

Journal of Chemical Physics

Link to article, DOI:

$10.1063 / 1.480566$

Publication date:

2000

Document Version

Publisher's PDF, also known as Version of record

Link back to DTU Orbit

Citation (APA):

Engel, V., \& Henriksen, N. E. (2000). Mapping of wave packets in direct fragmentation via pump-probe frequency integrated fluorescence spectroscopy. Journal of Chemical Physics, 112(1), 106-111. https://doi.org/10.1063/1.480566

\section{General rights}

Copyright and moral rights for the publications made accessible in the public portal are retained by the authors and/or other copyright owners and it is a condition of accessing publications that users recognise and abide by the legal requirements associated with these rights.

- Users may download and print one copy of any publication from the public portal for the purpose of private study or research.

- You may not further distribute the material or use it for any profit-making activity or commercial gain

- You may freely distribute the URL identifying the publication in the public portal 


\title{
Mapping of wave packets in direct fragmentation via pump-probe frequency integrated fluorescence spectroscopy
}

\author{
V. Engel ${ }^{\mathrm{a})}$ and N. E. Henriksen \\ Department of Chemistry, Technical University of Denmark, DTU 207, DK-2800 Lyngby, Denmark
}

(Received 27 July 1999; accepted 7 October 1999)

\begin{abstract}
We consider femtosecond excitation of a molecule to a dissociative electronic state. The quantum dynamics is recorded via delayed excitation to a higher electronic state and measurement of the total fluorescence from this state detected as a function of delay time. It is shown that the signal can be used to determine the probability density distribution of the outgoing wave packet describing the fragmentation. This, in particular, applies to the case of fragment detection since then the time-dependent signal directly measures the probability flux at a fixed value of the dissociation coordinate. Numerical examples illustrate the procedure. (C) 2000 American Institute of Physics. [S0021-9606(00)71101-9]
\end{abstract}

\section{INTRODUCTION}

The dynamics of elementary physical and chemical processes can be followed in real time by femtosecond pumpprobe spectroscopy. ${ }^{1-5}$ From these measurements one can, e.g., extract various characteristic times like the dissociation time of a chemical bond.

It has been shown recently that this technique also allows for the determination of probability density distributions as a function of time and internuclear distance. That is, the dynamics of wave packets can be mapped. To that end, time-frequency resolved emission spectroscopy, ${ }^{6}$ timeresolved photoelectron spectroscopy, ${ }^{7}$ time-resolved Coulomb explosion, ${ }^{8}$ and time-resolved time-of-flight fragment detection ${ }^{9}$ has been used. For example, time-resolved Coulomb explosion is a femtosecond pump-probe technique where the distribution of internuclear distances, at the time when the probe pulse ionizes the molecule, is inferred from the Coulomb-potential energy between the nuclei.

In any of the above methods, the signal has to be recorded as a function of delay-time and an energy. This is often experimentally demanding. The purpose of this paper is to show that it is possible to obtain the absolute square of molecular nuclear packets directly from integrated pumpprobe fluorescence spectroscopy, where the signal depends on delay-time only. The main result is that the timederivative of the signal is related to the absolute square of the wave function at a given point. The position of this point is, roughly, selected by the resonance condition for a given probe laser frequency. When, for example, direct photofragmentation is considered, the wave packet density in coordinate space describing the fragment motion can be constructed using the known asymptotic mean speed of the fragments.

This paper is organized as follows: In Sec. II, we summarize the theoretical approach to the calculation of integrated pump-probe fluorescence signals within the frame-

\footnotetext{
${ }^{a)}$ Permanent address: Institut für Physikalische Chemie, Universität Würzburg, Am Hubland, D-97074 Würzburg, Germany.
}

work of perturbation theory. We describe how the timederivative of the integrated pump-probe fluorescence signal is related to the probability flux and how this quantity is related to the absolute square of the wave function. Section III contains a numerical example for the direct dissociation of ICN into I $+\mathrm{CN}$. A perfect agreement is obtained between the wave packet extracted from the integrated pumpprobe signal and the actually calculated wave packet. Finally, a summary is given in Sec. IV.

\section{FLUX MAPPING PROCEDURE}

\section{A. Pump-probe signals}

The interaction of a molecule with two time-delayed femtosecond laser pulses is treated in what follows. Figure 1 displays the excitation scheme; potential curves as a function of a single coordinate $r$ are shown for two electronic states of a molecule. This one-dimensional picture is appropriate for diatomic molecules; in the case of larger systems, $r$ denotes the reaction coordinate. Starting from an initial wave function $\psi_{0}$ in the electronic ground state (0) the pump excitation prepares a time-dependent wave function in an excited electronic state (1) which can be written within first order perturbation theory as (atomic units are employed)

$$
\psi_{1}(t)=-i \int_{-\infty}^{t} d t^{\prime} U_{1}\left(t-t^{\prime}\right) W_{1}\left(t^{\prime}\right) U_{0}\left(t^{\prime}\right) \psi_{0}
$$

Here $U_{n}(t)$ is the time-evolution operator in the electronic state $(n)$ and the field-molecule interaction in the dipole approximation is given as

$$
W_{1}(t)=-\frac{1}{2} \mu_{01} f(t) e^{-i \omega_{1} t} .
$$

$f(t)$ denotes a pulse envelope, $\omega_{1}$ the frequency, and $\mu_{01}$ is the projection of the transition dipole moment on the polarization of the electric field. Only the term leading to absorption is considered. 


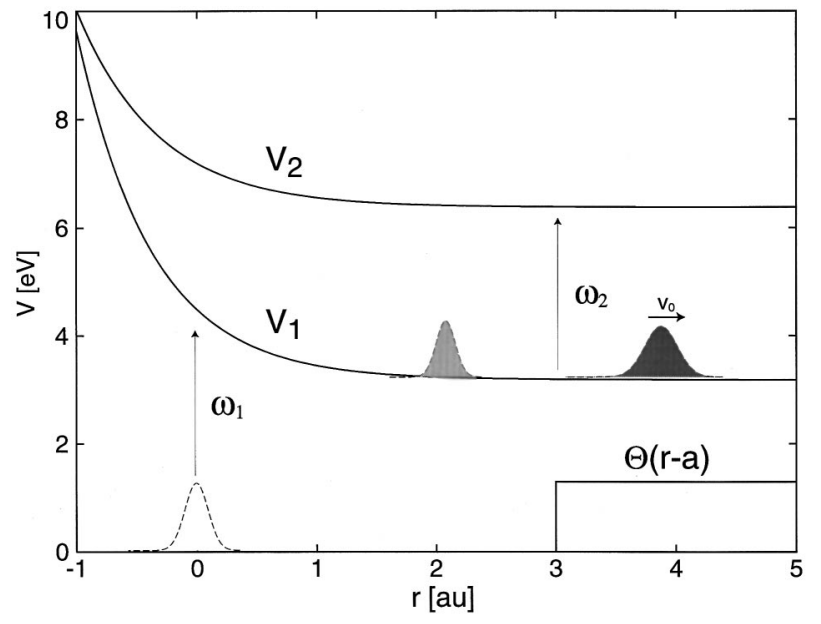

FIG. 1. Pump-probe scheme for direct dissociation; the pump pulse $\left(\omega_{1}\right)$ prepares a wave packet in an excited electronic state with potential curve $V_{1}$. The probe step $\left(\omega_{2}\right)$ induces a transition to another electronic state $\left(V_{2}\right)$ and the total fluorescence from this state is measured. The probe-laser frequency is chosen such, that the wave packet is probed when it enters the asymptotic region where the distance $r$ exceeds a critical point $a$. This spatial window is denoted as $\Theta(r-a)$. The potentials represent a simple model for ICN dissociation where $r$ is the I-C distance relative to the equilibrium position in the electronic ground state.

The probe process is initiated by a second pulse delayed by a time $T$, and a wave function in the electronic state (2) is created. Using again first order perturbation theory, this function takes the form,

$$
\psi_{2}(t)=-i \int_{-\infty}^{t} d t^{\prime} U_{2}\left(t-t^{\prime}\right) W_{2}\left(t^{\prime}-T\right) \psi_{1}\left(t^{\prime}\right),
$$

where $\psi_{1}(t)$ is given by Eq. (1). The interaction energy is

$$
W_{2}(t-T)=-\frac{1}{2} \mu_{12} f(t-T) e^{-i \omega_{2}(t-T)} .
$$

Here the notation of the different quantities is analogous as above. The total pump-probe fluorescence signal $P(T)$ is assumed to be proportional to the norm of $\psi_{2}(t)$ after the second pulse decayed to zero,

$$
P(T)=\lim _{t \rightarrow \infty} \int d r\left|\psi_{2}(r, t)\right|^{2} .
$$

If one neglects commutators between the kinetic energy operators and the potentials appearing in the propagators, the signal can be written as ${ }^{10-14}$

$$
P(T)=\int d r\left|F\left(D(r)-\omega_{2}\right)\right|^{2}\left|\psi_{1}(r, T)\right|^{2},
$$

where we used the definition

$$
F\left(D(r)-\omega_{2}\right)=\int_{-\infty}^{+\infty} d t f(t) e^{i\left(D(r)-\omega_{2}\right) t}
$$

and $D(r)=V_{2}(r)-V_{1}(r)$ is the difference between the potential energy curves in the electronic states (2) and (1). Thus, the signal measures the norm of the wave packet $\psi_{1}$ at time $T$ within the window $F\left(D(r)-\omega_{2}\right)$ (Franck-Condon window). The latter is centered around the region where the laser frequency equals the difference potential $D(r)$.

\section{B. Flux mapping}

Let us discuss the case of fragment detection for a dissociation process. Here the carrier frequency of the probe laser is tuned to match the asymptotic value of the difference potential $(D(r=\infty)=\Delta)$. Then the Franck-Condon window extends from a critical distance $a$ to infinity (see Fig. 1). Naturally, since the ultrashort probe pulse is spectrally broad this distance is defined within the energy uncertainty of the pulse only. The pump-probe signal becomes different from zero if the wave packet $\psi_{1}$ passes the distance $a$.

To a good approximation, we can replace the FranckCondon window by a Theta function, $F\left(D(r)-\omega_{2}\right)$ $=\theta(r-a)$, so that the signal becomes

$$
P(T)=\int_{a}^{\infty} d r\left|\psi_{1}(r, T)\right|^{2} .
$$

We now show that the integrated signal can be used to obtain the probability density distribution $\rho_{1}(r, T)=\left|\psi_{1}(r, T)\right|^{2}$. Therefore we calculate the time-derivative of the signal to obtain

$$
\frac{d}{d T} P(T)=\int_{a}^{\infty} d r \psi_{1}^{*}(r, T) \frac{d}{d T} \psi_{1}(r, T)+\text { c.c. },
$$

where c.c. denotes the complex conjugate. Inserting the firstorder expression from Eq. (1) yields

$$
\begin{aligned}
\frac{d}{d T} P(T)= & \int_{a}^{\infty} d r \psi_{1}^{*}(r, T)\left(-i H_{1} \psi_{1}(r, T)\right. \\
& \left.-i W_{1}(T) U_{0}(T) \psi_{0}(r)\right)+ \text { c.c. }
\end{aligned}
$$

The second term vanishes exactly for nonoverlapping pump - and probe-pulses and is most likely to be zero since the critical distance $a$ in general will not fall into the FranckCondon region for the pump excitation (see Fig. 1). Furthermore for $r>a$ the excited state Hamiltonian $H_{1}$ equals the kinetic-energy operator except for a constant which does not change the following expressions. Thus we arrive at the equation

$$
\frac{d}{d T} P(T)=\frac{i}{2 m} \int_{a}^{\infty} d r \psi_{1}^{*}(r, T) \frac{d^{2}}{d r^{2}} \psi_{1}(r, T)+\text { c.c. },
$$

$m$ being a mass. The integral can now be evaluated to give the final expression for the time-derivative of the pumpprobe signal,

$$
\begin{aligned}
\frac{d}{d T} P(T)= & \frac{-i}{2 m}\left\{\psi_{1}^{*}(r, T) \frac{d}{d r} \psi_{1}(r, T)\right. \\
& \left.-\psi_{1}(r, T) \frac{d}{d r} \psi_{1}^{*}(r, T)\right\}_{a}=j(a, T),
\end{aligned}
$$

where the subscript indicates that the expression has to be evaluated at $r=a$. Thus, the time-derivative of the signal equals the probability flux $j(a, T)$ through the point $r=a$. Although $(d / d T) P(T)$ does not directly reflect the probability density at a certain point in time, it is very much related to it. Writing the wave function in terms of the density $\rho_{1},{ }^{15}$

$$
\psi_{1}(r, T)=\sqrt{\rho_{1}(r, T)} e^{i S(r, T)}
$$


with a real function $S(r, T)$, the signal takes the form,

$$
\frac{d}{d T} P(T)=\frac{1}{m}\left\{\rho_{1}(r, T) \frac{d}{d r} S(r, T)\right\}_{a} .
$$

If the function $S(r, T)$ depends only weakly on time, the signal indeed is proportional to the probability density passing through $a$.

To get some insight into the time-dependence of $S(r, T)$, it is instructive to regard the motion of a free Gaussian wave packet which can be written as

$$
\psi_{G}(r, t)=e^{i A_{t}\left(r-r_{t}\right)^{2}+i p_{0}\left(r-r_{t}\right)+i s_{t}}
$$

where $A_{t}$ and $s_{t}$ are complex parameters, $p_{0}$ is the mean momentum, and $r_{t}$ is the center of the Gaussian. If we neglect the real part of $A_{t}$ the function $(d / d r) S$ equals $p_{0}$, and indeed it does not depend on time. Then the flux has the interpretation of being the product of the probability density times the mean velocity. ${ }^{15}$ In more detail, if at time $t=t_{0}$ the parameter $A_{t}$ is equal to $A_{0}$, then its time-dependence is given as ${ }^{16}$

$$
A_{t}=\frac{A_{0}}{1+\left(2 A_{0} / m\right)\left(t-t_{0}\right)} .
$$

The time needed for the pump-probe signal to increase from zero to a constant value approximately equals the time it takes a wave packet to pass a distance corresponding to its width $\Delta r_{0}$ at time $t_{0}$. Replacing $\left(t-t_{0}\right)$ by $\Delta r_{0} / \mathrm{v}_{0}$ and rewriting the velocity in terms of the available energy $E$, one finds

$$
A_{t}=\frac{A_{0}}{1+\left(2 A_{0} \Delta r_{0}\right) /(\sqrt{2 m E})} .
$$

It then follows that the time-dependence of $A_{t}$ is weak for large mass and available energy. The above connection enables us to determine $\rho_{1}$ as a function of distance as will be demonstrated in Sec. III, using a numerical example.

Let us, at this point, comment on the general, multidimensional ( $N$-dimensional) case. Here the mapping is, of course, not unique in the sense that a signal recorded as a function of one parameter cannot reflect a density depending on many coordinates. In the above equations now all the spatial integrals become multiple integrals. The critical distance $a$ has to be replaced by a hypersurface. The timederivative of the signal measures the flux into this surface. However, if the concept of a reaction coordinate holds, the $N-1$ dimensions corresponding to bound degrees of freedom can be integrated out and the flux-mapping procedure will give, to a good approximation, a cut of the moving wave packet along this coordinate.

Let us, for completeness, discuss the detection of the wave packet in the inner potential region (transition-state detection). In this case the probe frequency is chosen such that the Franck-Condon window is located at smaller distances, so that the probe excitation takes place at times before the wave packet $\psi_{1}$ reaches the asymptotic region. To a good approximation, we can replace the window function $F\left(D(r)-\omega_{2}\right)$ by a function $\theta(r-(a-d)) \theta((a+d)-r)$ with a width $2 d$. Following the same manipulations as above, the time derivative of the pump-probe signal takes the form

$$
\frac{d}{d T} P(T)=j(a-d, T)-j(a+d, T) .
$$

Here we assumed that the potential $V_{1}(r)$ is equal at the points $a$ and $b$, since otherwise an additional term is to be considered [see Eq. (10)]. Thus, we measure the difference of fluxes at two spatial points for a given time and it is not obvious how to invert the density $\rho_{1}(r, t)$. However, if the points $a$ and $b$ are separated by a sufficiently small distance, that is, the Franck-Condon window is small, we may write

$$
\begin{aligned}
\frac{d}{d T} P(T) & \sim(2 d) \frac{j(a-d, T)-j(a+d, T)}{2 d} \\
& \sim-(2 d)\left\{\frac{d}{d r} j(r, T)\right\}_{a}=(2 d) \frac{d}{d T} \rho_{1}(a, T),
\end{aligned}
$$

where the last equality follows from the continuity equation satisfied by $\rho_{1}(r, t) .{ }^{15}$ Integrating over time one finds the equation,

$$
P(T) \sim(2 d) \rho_{1}(a, T),
$$

which alternatively can be obtained directly from Eq. (6) within the same approximations. Thus, in the case of a transition-state detection and for a narrow Franck-Condon window, the density can be directly correlated to the pumpprobe signal.

\section{NUMERICAL EXAMPLE}

\section{A. Model}

The dissociation of ICN into I $+\mathrm{CN}$ will be chosen as a numerical example. In the first gas phase femtosecond timeresolved experiments, Zewail and co-workers investigated this system. ${ }^{17,18}$ Quantum calculations were performed within a one-dimensional model by Williams and Imre ${ }^{19}$ and a good overall agreement was found with the data. Later it was shown by Heather and Metiu ${ }^{20}$ that the rotational degrees of freedom have to be considered to explain finer details of the experiment. Here we use the simple model of Williams and Imre. The employed potential curves are displayed in Fig. 1 as a function of the I-C separation using the equilibrium distance in the electronic ground state as the origin of the axis. The origin of the energy scale corresponds to the eigenenergy of the initial wavefunction $\psi_{0}$ in the electronic ground state. The initial state was taken to be a Gaussian centered at $r=0$ with a width (full-width at halfmaximum) of 0.1 a.u. We solve the time-dependent Schrödinger equation with the split-operator method ${ }^{21}$ and the time-integrals which appear in perturbation theory are calculated as described in Ref. 22. The transition dipole moments were set to unity in all calculations and Gaussian envelope functions for the pump pulse were employed throughout. 


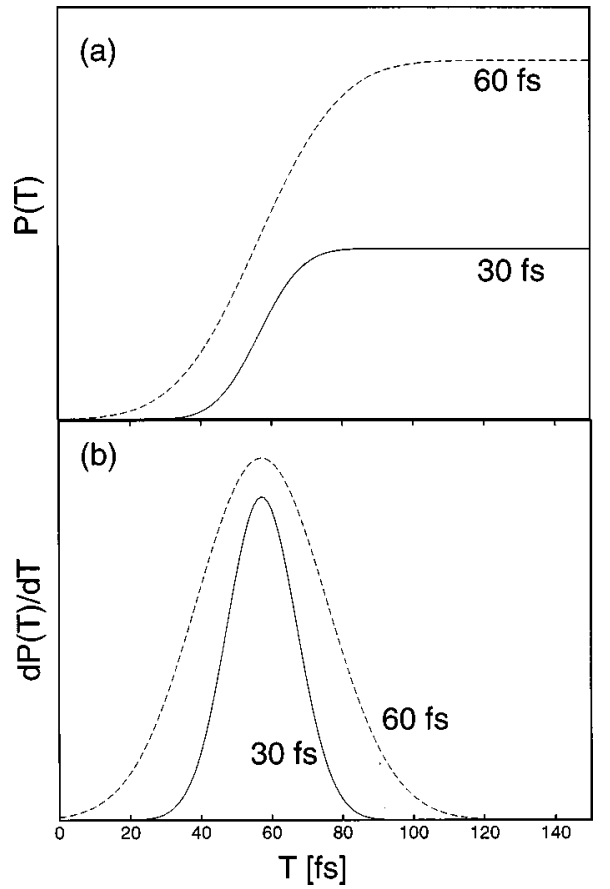

FIG. 2. Panel (a) shows the calculated pump-probe signal $P(T)$ for pump pulses of different width, as indicated. Panel (b) contains the time-derivative of $P(T)$. The zero of the time-scale corresponds to the center of the respective pump pulse.

\section{B. Flux mapping}

Figure 2(a) displays pump-probe signals calculated for two different widths (full-width at half-maximum) of the pump laser, as indicated. Here the simple expression in Eq. (8) for the signal and a distance of $a=3$ a.u. was employed. The frequency was set to $4.6 \mathrm{eV}$ which corresponds to excitation in the vicinity of the absorption maximum. In both cases the time $T=0$ corresponds to the maximum of the respective pump pulse. The typical increase from zero to a constant is seen which reflects the motion of the prepared wave packet into the excitation window. The particular form of the function can be understood analytically using a generalized Gaussian wave packet for $\psi_{1}(r, T) .^{23}$

The lower panel shows the time-derivative of the signal. In both cases one finds a Gaussian-type curve which is broader for the longer pump pulse. We now will map these curves into coordinate space using the procedure proposed in Sec. II B. Since the wave packets are located in the asymptotic region they move with a constant speed towards larger distances. We assume the expectation value of the energy to be $\left\langle\psi_{1}\left|H_{1}\right| \psi_{1}\right\rangle=\omega_{1}-V_{1}(r=\infty)$ which reflects energy conservation in the limit of long pulses. Then the velocity $\mathrm{V}_{0}$ is given by $\mathrm{V}_{0}=\sqrt{2\left(\omega_{1}-V_{1}(r=\infty)\right) / m}$, where $m$ is the reduced mass of the I-CN system. Using this value the $r$ dependent density can be obtained as

$$
\rho_{1}\left(a+\mathrm{v}_{0} t, T\right) \sim\left\{\frac{d}{d \lambda} P(\lambda)\right\}_{\lambda=T-t} .
$$

In performing the construction of the wave-packet density it should be clear that in order to obtain it in its entirety, the wave packet must have passed completely the point $a$.

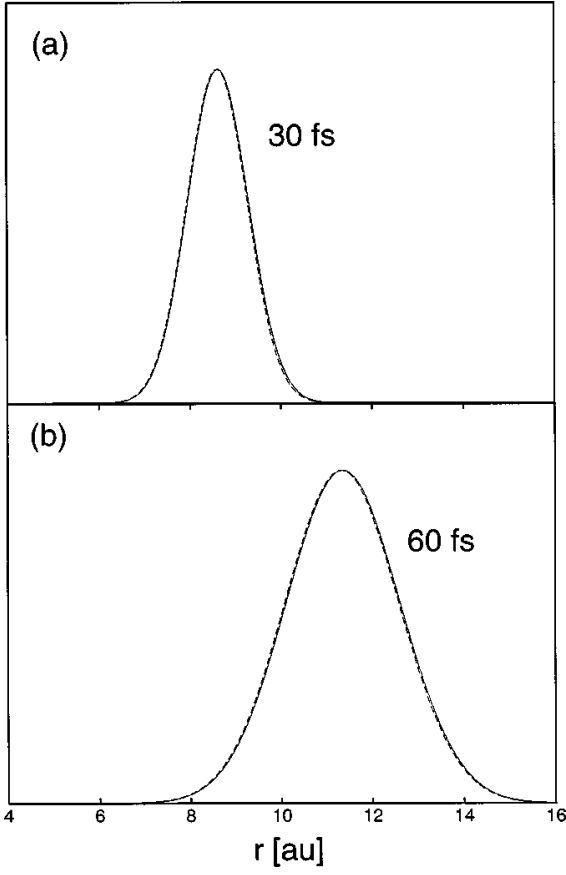

FIG. 3. Comparison of the absolute square of the wave function obtained from the time-propagation (solid line) and from the flux-mapping procedure (dashed line). The respective curves were normalized to assume the same value at their maximum. Functions for pulses of 30 (a) and $60 \mathrm{fs}$ (b) are shown at different times.

Figure 3 compares the actually calculated densities $\rho_{1}(r, t)$ with the densities obtained via Eq. (21). Here we used pulses of 30 (60) fs width and Eq. (21) was applied 140 (180) fs after the maximum of the respective pump-pulse. The different curves for either pulse length are hardly distinguishable. This confirms that indeed the probability density amplitudes can be inverted from the pump-probe fluorescence signal and the time-dependence of the phase function $(d / d r) S(r, T)$ is neglegible in our example.

We note that the outgoing wave packet spreads in the coordinate space. The spreading cannot be determined from the signal. However, this would in principle be possible if excitation to another excited electronic state at a different critical distance $a$ could be achieved. Furthermore the distance $a$ is, in general, not precisely known. In this case the $r$-dependent density can be determined from an experimental signal within an unknown shift on the $r$-axis only.

\section{SUMMARY}

As the main result of the present paper we have shown that it is possible to invert probability densities $\rho_{1}(r, T)$ from frequency integrated time-resolved pump-probe fluorescence signals. If the probe laser frequency is chosen to induce transitions in the dissociation products, the timederivative of the signal equals the probability flux through the critical distance $a$, where the interaction potential between the fragments is close to zero. Since this flux is proportional to $\rho_{1}(a, T)$, one is able to construct the density as a function of the reaction coordinate $r$. If the distance $a$ is 

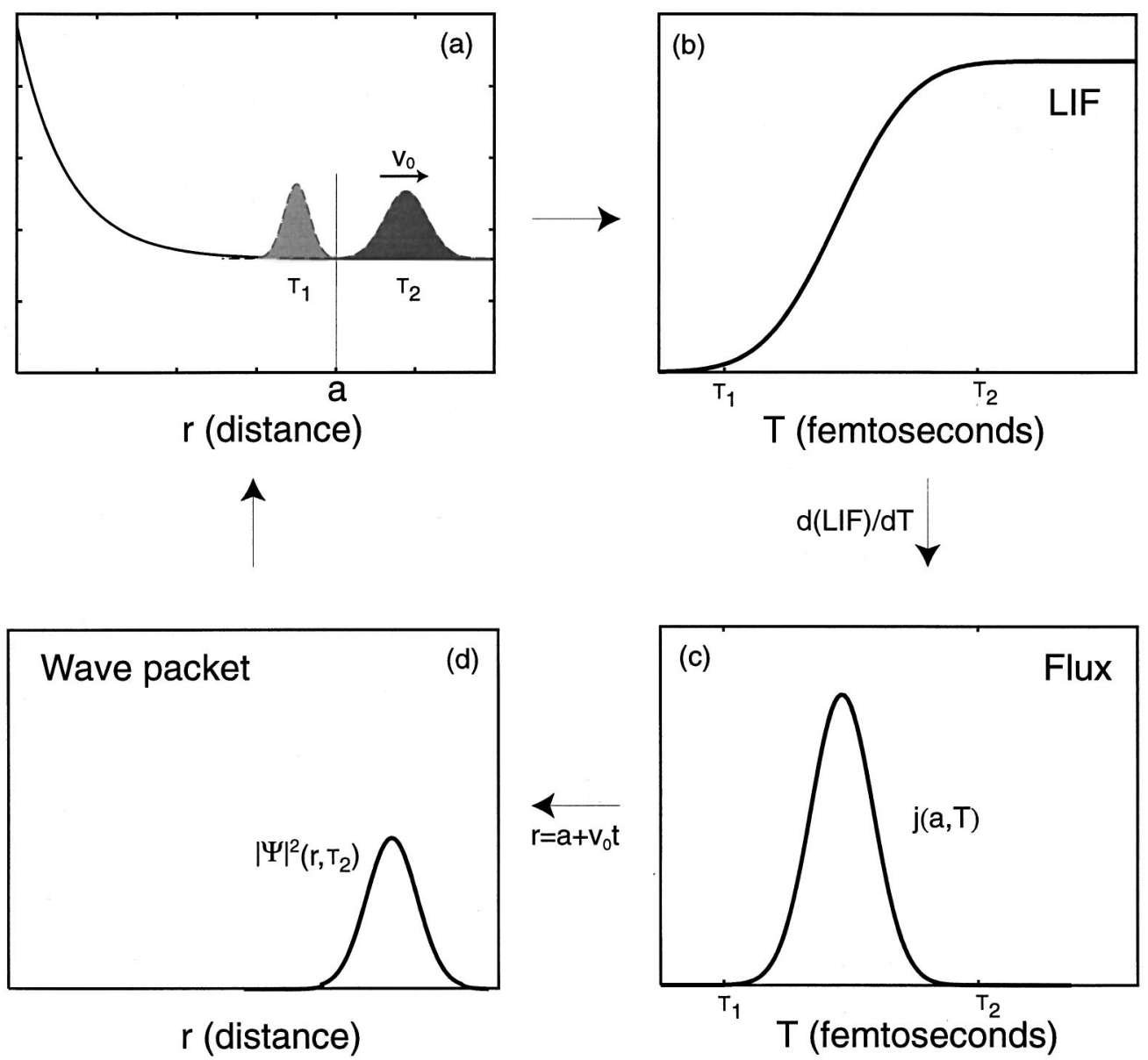

FIG. 4. Illustration of the flux-mapping procedure for fragment detection in direct photodissociation. The motion of a wave packet into the asymptotic region (a) with velocity $\mathrm{v}_{0}$ gives rise to the pump-probe signal LIF (b). The time-derivative of this signal (c) equals the flux through the critical point $a$. This flux can be used to construct the absolute square of the wave packet via a linear transformation.

known this is possible on an absolute scale, otherwise the density will be shifted by an unknown amount on the coordinate scale.

The flux mapping procedure is illustrated in Fig. 4; the wave packet prepared in the pump process enters the detection window starting at distance $a$ at time $T_{1}$ [panel (a)]. Moving with a constant speed $\mathrm{v}_{0}$, it is completely located within the window at time $T_{2}$. This gives rise to the increase of the pump-probe signal shown in panel (b). The timederivative of the signal is equal to the flux through the point $a$ [panel (c)]. The latter is proportional to the absolute square of the wave packet which allows for a construction of the density using a linear transformation [panel (d)].

In the case of transition-state detection, a similar construction of the probability density is possible. This, however, rests on the assumption that the Franck-Condon window for the probe excitation is sufficiently narrow.

The present procedure does not require the recording of a two-dimensional signal to construct the wave-packet density as is necessary in other methods. However, the application of the method to purely bound state motion is limited and its strength lies in the construction of wave-packet densities for fragment motion in the case of direct dissociation.

\section{ACKNOWLEDGMENTS}

Financial support by the Deutsche Forschungsgemeinschaft is gratefully acknowledged. V.E. thanks the Technical University of Denmark at Lyngby for the opportunity to participate in its program for visiting professors and for the hospitality during his visit at the Department of Chemistry.

${ }^{1}$ A. H. Zewail, Femtochemistry (World Scientific, Singapore, 1994), Vols. 1 and 2.

${ }^{2}$ Femtosecond Chemistry, edited by J. Manz and L. Wöste (VCH, Weinheim, 1995).

${ }^{3}$ Femtochemistry, edited by M. Chergui (World Scientific, Singapore, 1996).

${ }^{4}$ Femtochemistry and Femtobiology, edited by V. Sundström (Imperial College Press, London, 1997).

${ }^{5}$ Chemical Reactions and Their Control on the Femtosecond Time Scale, edited by P. Gaspard and I. Burghardt (Wiley, New York, 1997).

${ }^{6}$ T. J. Dunn, J. N. Sweetser, I. A. Walmsley, and C. Radzewicz, Phys. Rev. Lett. 70, 3388 (1993).

${ }^{7}$ A. Assion, M. Geisler, J. Helbing, V. Seyfried, and T. Baumert, Phys. Rev. A 54, R4605 (1996).

${ }^{8}$ P. B. Corkum, M. Y. Ivanov, and J. S. Wright, Annu. Rev. Phys. Chem. 48, 387 (1997).

${ }^{9}$ A. Assion, T. Baumert, M. Geisler, V. Seyfried, and G. Gerber, Eur. Phys. J. D 4, 145 (1998). 
${ }^{10}$ M. Braun, C. Meier, and V. Engel, J. Chem. Phys. 103, 7907 (1995).

${ }^{11}$ Z. Li, J.-Y. Fang, and C. C. Martens, J. Chem. Phys. 104, 6919 (1996).

${ }^{12}$ E. M. Hiller and J. A. Cina, J. Chem. Phys. 105, 3419 (1996).

${ }^{13}$ J. Cao and K. R. Wilson, J. Chem. Phys. 106, 5062 (1997).

${ }^{14}$ H. Dietz and V. Engel, J. Phys. Chem. A 102, 7406 (1998).

${ }^{15}$ J. J. Sakurai, Modern Quantum Mechanics (Addison-Wesley, Redwood City, 1985).

${ }^{16}$ E. J. Heller, J. Chem. Phys. 62, 1544 (1975).
${ }^{17}$ M. Dantus, M. J. Rosker, and A. H. Zewail, J. Chem. Phys. 87, 2395 (1987).

${ }^{18}$ M. J. Rosker, M. Dantus, and A. H. Zewail, Science 241, 1200 (1988).

${ }^{19}$ S. O. Williams and D. G. Imre, J. Phys. Chem. 92, 6648 (1988).

${ }^{20}$ R. Heather and H. Metiu, Chem. Phys. Lett. 157, 505 (1989).

${ }^{21}$ M. D. Feit, J. A. Fleck, and A. Steiger, J. Comput. Phys. 47, 412 (1982).

${ }^{22}$ V. Engel, Comput. Phys. Commun. 63, 228 (1991).

${ }^{23}$ N. E. Henriksen, Adv. Chem. Phys. 91, 433 (1995). 